\title{
Spatially and temporally fluctuating selection at non-MHC immune genes: evidence from TAP polymorphism in populations of brown trout (Salmo trutta, L.)
}

\author{
LF Jensen ${ }^{1,2}$, MM Hansen ${ }^{2}$, K-LD Mensberg ${ }^{2}$ and V Loeschcke ${ }^{1}$ \\ ${ }^{1}$ Department of Ecology and Genetics, University of Aarhus, Aarhus C, Denmark and ${ }^{2}$ Department of Inland Fisheries, Danish Institute \\ for Fisheries Research, Silkeborg, Denmark
}

\begin{abstract}
Temporal samples of Danish brown trout (Salmo trutta) from populations representing varying geographical scales were analysed using eight putatively neutral microsatellite loci and two microsatellite loci embedded in TAP genes (Transporter associated with Antigen Processing). These genes encode molecules that are central to the major histocompatibility complex $(\mathrm{MHC})$ class I restricted antigen presentation and thus integral components in the adaptive immune system. As such, they could be influenced by selection, driven by pathogens and parasites in a manner similar to $M H C$ genes. Analysis of allele frequencies at presumably neutral microsatellite loci revealed a temporally unstable population
\end{abstract}

structure within regions, while the population structure was stable over time among regions. Analyses of the two TAP markers indicated an effect of selection at both a regional and micro-geographical spatial scale. Moreover, signals of divergent selection among temporal samples within localities suggest that selection also might fluctuate at a temporal scale. These results suggest that immune genes other than the classical $M H C$ class I and II might be subject to selection and warrant further studies of functional polymorphism of such genes in natural populations.

Heredity (2008) 100, 79-91; doi:10.1038/sj.hdy.6801067; published online 7 November 2007

Keywords: adaptive variation; hitch-hiking selection; major histocompatibility complex genes; microsatellite DNA; spatiotemporal genetic variation; transporter associated with antigen-processing genes

\section{Introduction}

Analysis of neutral genetic markers such as microsatellite DNA provides insights into the role of migration, drift and mutation in shaping the genetic structure of populations. However, neutral markers do not provide direct information about the possible adaptive significance of genetic differentiation, because neutral variation does not necessarily correlate with variation under influence of selection (Merilä and Crnokrak, 2001; McKay and Latta, 2002). Obviously therefore, genes known to be influenced by natural selection could provide much more direct information on adaptive divergence among local populations. The classical major histocompatibility complex $(\mathrm{MHC})$ class I and II loci offer a particularly promising avenue to this approach (Bernatchez and Landry, 2003), due to the extensive knowledge on the structure and function of their encoded molecules and strong evidence for natural selection at these loci, for example increased rates of non-synonymous nucleotide substitutions, high levels of polymorphism and allele frequency distributions that are

Correspondence: Dr LF Jensen, Department of Ecology and Genetics, University of Aarhus, Building 540, Ny Munkegade, Aarhus C DK-8000, Denmark.

E-mail: lasse.fast@gmail.com

Received 25 May 2007; revised 30 August 2007; accepted 31 August 2007; published online 7 November 2007 more even than expected under neutrality (reviewed by Bernatchez and Landry, 2003; Piertney and Oliver, 2006). The parasites and pathogens in the environment are suspected to be the responsible selective agents, due to the role of the $\mathrm{MHC}$ molecules in presenting foreign polypeptides to the cytotoxic $\mathrm{T}$ cells of the immune system, thereby facilitating the flow of communication between the infected cell and the effector cells of the immune system.

Balancing selection through heterozygote advantage (Doherty and Zinkernagel, 1975; Black and Salzano, 1981) or rare allele advantage (Bodmer, 1972; Slade and McCallum, 1992) have been the favoured selection models to explain polymorphism at MHC genes. Yet, there is little empirical evidence favouring one model over the other and models of selection acting at $\mathrm{MHC}$ genes have been widely debated (Maruyama and Nei, 1981; Takahata and Nei, 1990; Hughes and Nei, 1992; Slade and McCallum, 1992; Borghans et al., 2004; De Boer et al., 2004). A third selection model, proposed by Hedrick et al. (1987) and later modified by Hedrick (2002) (but see also Meyer and Thomson, 2001), suggests that fluctuating selection in time and space is sufficient to maintain realistic levels of polymorphism at immune genes. In this model, fitness values constantly change as a function of pathogen frequency.

Although Trowsdale (1995) labelled MHC molecules 'the centre of the immune universe', the relative contribution from $M H C$ to the immune response is rather small 
(Jepson et al., 1997). Thus, a number of non-MHC genes contribute significantly to variation in the immune response and hence might also be subject to selection. These genes include loci encoding the Transporter associated with Antigen Processing (TAP). Before endogenously derived peptides, for example, some bacteria and virus are displayed (by $M H C$ class I molecules) to the effector cells of the immune system, they are degraded and subsequently actively transported to the lumen of the rough endoplasmic reticulum. Here the association with the $M H C$ class I molecules takes place. The transport of peptides across the membrane of the endoplasmic reticulum is mediated by the TAP molecules. These molecules are heterodimeric proteins composed of one TAP1 and one TAP2 subunit each encoded by separate loci located within the $\mathrm{MHC}$ region. In salmonids, Grimholt et al. (2002) identified two TAP2 loci denoting these TAP $2 A$ and $T A P 2 B$ respectively. Functional polymorphism has been observed in TAP molecules (Monaco, 1992; Powis et al., 1992; Heemels and Ploegh, 1994), as evidenced by differences among the allelic products in the ability to transport specific peptides. Moreover, the binding regions of the MHC class I and TAP molecules share similarities in their specificity to C-terminal residues of the processed peptides and it has been suggested that these molecules have coevolved (Abele and Tampé, 2006). Furthermore, particular TAP alleles have been associated with susceptibility or resistance to a number of infectious diseases in human (Rajalingam et al., 1997; Kuzushita et al., 1999; Gomez et al., 2006) and several immune evasion mechanisms directed at the TAP molecule have been adopted by viruses (Abele and Tampé, 2006). These findings stress the importance of the TAP molecules in the function of the immune system and suggest that, in addition to genetic variation at MHC class I and II genes, selection driven by parasites and pathogens may be acting on the TAP genes as well. While polymorphism at the MHC genes has received much attention in molecular evolutionary and ecological research, investigation of polymorphism at TAP genes has attracted much less interest.

The tendency of salmonid fishes to form local populations inhabiting different environments is expected to promote evolutionary changes (Taylor, 1991). Several studies of brown trout (Salmo trutta) populations, using both allozymes (Ferguson, 1989), mitochondrial DNA (Laikre et al., 2002) and microsatellites (Hansen et al., 2002) have shown that a considerable proportion of the genetic variation is distributed among populations. However, little is known about the adaptive significance of this genetic differentiation and the geographical scale at which local adaptation might occur. Hansen et al. (2002) used empirical estimates of gene flow and effective population size to make a theoretically based evaluation of the likelihood of local adaptation, concluding that local adaptations could indeed persist in brown trout populations, especially at a regional scale encompassing several river systems.

Here we analyse genetic variation at two microsatellite markers linked to the TAP $2 A$ and $T A P 2 B$ loci, respectively, to investigate if any of these loci might be influenced by selection and therefore deserve further studies in this respect. Specifically we study the spatiotemporal distribution of genetic variation in brown trout
(Salmo trutta) at the two TAP2-linked loci and a set of presumably neutral microsatellite loci and investigate whether these sets of markers are subject to the same evolutionary forces. In other words does polymorphism at TAP2 markers seem to evolve neutrally in natural populations? Using a hierarchical experimental design we address this issue at (i) a temporal scale, (ii) a microgeographical scale and (iii) at a regional scale.

\section{Materials and methods}

\section{The studied localities}

The study was based on samples from three geographical regions in Jutland, Denmark: the Odderaa River system, the Gudenaa River system and the Lake Hald system (see Figure 1 and Table 1), all of which are unaffected by stocking activities. The Odderaa River system, which is inhabited by presumably interbreeding resident and anadromous brown trout (Salmo trutta), was sampled by electrofishing in 1992 and 2004. Adipose fin clips were collected from the same localities in both the years: Asbæk (ASBK), Kragebæk (KRAG), Fiskbæk (FISK), Stampemølle bæk (STAM) and Assedrup bæk (ASSE), all first-order tributaries, and two localities within the main river located approximately $4 \mathrm{~km}$ apart (ODR1 and ODR2).

The Gudenaa River system was represented by two sampled tributaries. The River Tjærbæk was sampled in 1994 at two locations, located above (TO94) and below (TN94) an impassable barrier, whereas River Brandstrup Bæk (BR95) was sampled at one location in 1995. All localities contain populations of both resident and anadromous brown trout, except the upstream part of the River Tjærbæk (TN94), which only contains resident trout.

The small rivers Kapeldal Bæk (KAP) and Krobæk (KRO), both flowing into Lake Hald, Central Jutland, were sampled in 1989 and 2002. Both rivers contain populations of brown trout migrating into the lake. The two rivers are separated by a waterway distance of approximately $2 \mathrm{~km}$. The Lake Hald system is a part of the Gudenaa River drainage but has been completely isolated from allochthonous gene flow since the 12-14th century due to an impassable dam in the outlet of the lake.

\section{Neutral microsatellite loci}

DNA was extracted using either the phenol-chloroform protocol by Taggart et al. (1992) or the Chelex resin method described by Estoup et al. (1996). Eight presumably neutral microsatellite loci were PCR amplified: Str15, Str60, Str73 (Estoup et al., 1993), Ssosl 417 (Slettan et al., 1995), Ssosl 438 (Slettan et al., 1996), Ssa 85, Ssa 197 (O'Reilly et al., 1996) and T3-13 (Estoup et al., 1998). PCR conditions were as described in Hansen (2002). The amplified microsatellite loci were analysed on a Pharmacia ALFexpress automated sequencer (Uppsala, Sweden) according to recommendations by the manufacturer (data are provided in appendix).

\section{TAP2-linked microsatellite loci}

Two microsatellite loci located in intron 5 of the SasaTAP $2 A$ and Sasa-TAP2B loci, respectively, were PCR amplified using the primers by Grimholt et al. (2002). In 


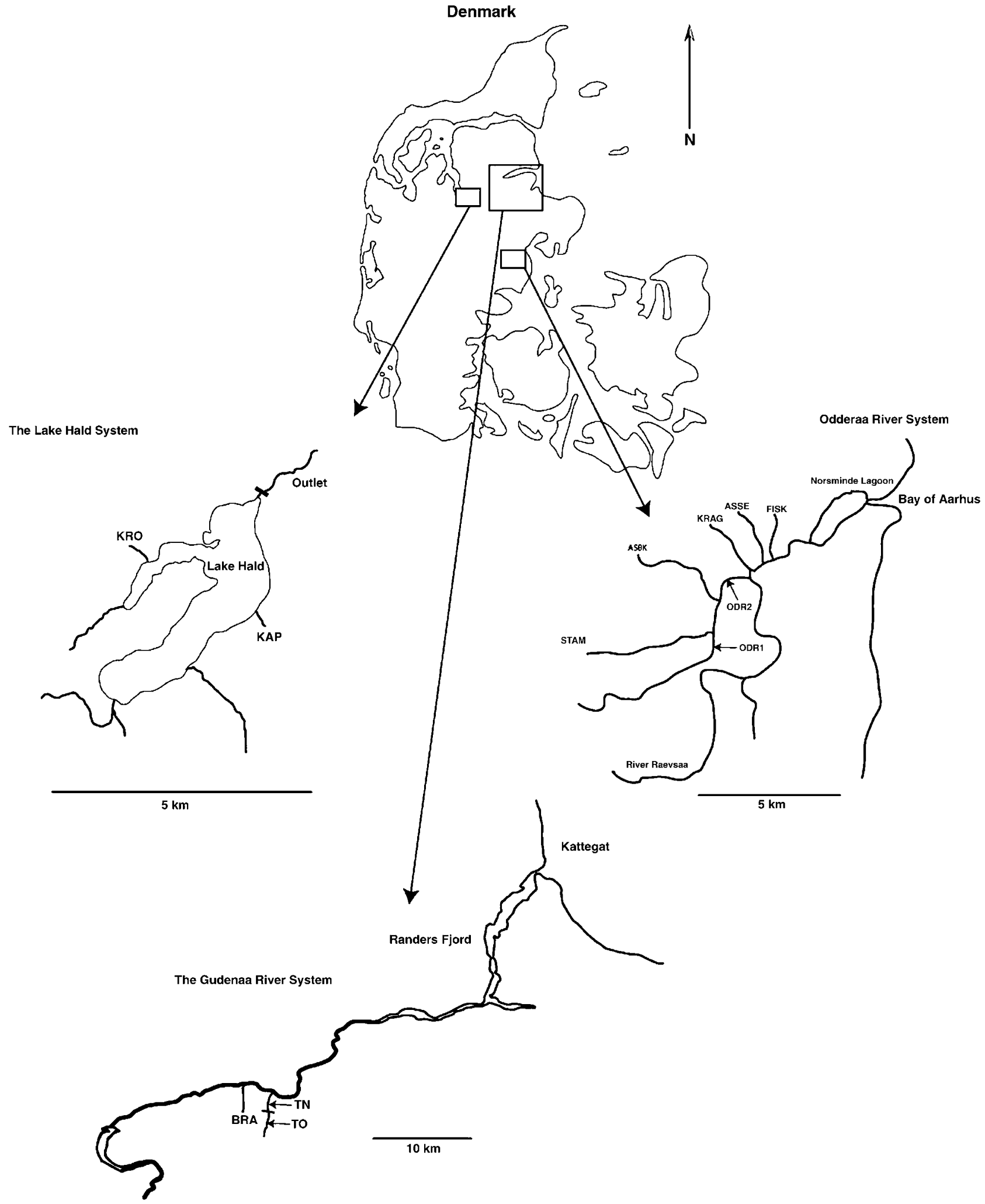

Figure 1 Map showing the geographical location of the localities sampled and the location of drainages within Denmark.

Atlantic salmon, the TAP2B locus has been found to map to the major MHC class I antigen UBA (Grimholt et al., 2002). This association also seems to be present in the brown trout populations analysed in the present study as previous studies have found linkage between the $T A P 2 B$ marker and a microsatellite marker located in an untranscribed region of UBA (LF Jensen, unpublished data). The UBA marker was not analysed in the present 
Table 1 Sample information

\begin{tabular}{lccc}
\hline Locality & $\begin{array}{c}\text { Sampling } \\
\text { year }\end{array}$ & $\begin{array}{c}\text { Sample } \\
\text { abbreviation }\end{array}$ & $\begin{array}{c}\text { Sample } \\
\text { size }\end{array}$ \\
\hline $\begin{array}{l}\text { The Odderaa River system } \\
\text { River Asbæk }\end{array}$ & 1992 & ASBK92 & 59 \\
River Kragebæk & 2004 & ASBK04 & 50 \\
& 1992 & KRAG92 & 31 \\
River Fiskbæk & 2004 & KRAG04 & 49 \\
River Stampemølle bæk & 1992 & FISK92 & 32 \\
& 2004 & FISK04 & 54 \\
River Assedrup bæk & 1992 & STAM92 & 29 \\
& 2004 & STAM04 & 50 \\
River Odderaa, locality 1 & 1992 & ASSE92 & 33 \\
& 2004 & ASSE04 & 49 \\
River Odderaa, locality 2 & 2004 & ODR1-92 & 30 \\
& 1992 & ODR1-04 & 50 \\
ODR2-92 & 35 \\
The Lake Hald system & 2004 & ODR2-04 & 50 \\
River Krobæk & & & \\
River Kapeldalbæk & 1989 & KRO89 & 83 \\
& 2002 & KRO02 & 43 \\
The Gudenaa River system & 1989 & KAP89 & 22 \\
River Tjærbæk, & 2002 & KAP02 & 41 \\
above dam & & & \\
River Tjærbæk, & 1994 & TO94 & 47 \\
below dam & 1994 & TN94 & 49 \\
River Brandstrup bæk & 1995 & BRA95 & 59 \\
\hline
\end{tabular}

study due to the presence of null alleles at this locus. The TAP2A locus belongs to a different linkage group (Grimholt et al., 2002). The PCR profile for the two markers was an initial denaturing stage of $5 \mathrm{~min}$ at $94{ }^{\circ} \mathrm{C}$ followed by 30 cycles of $94{ }^{\circ} \mathrm{C}$ for $30 \mathrm{~s}, 54{ }^{\circ} \mathrm{C}$ for $30 \mathrm{~s}$ and $72{ }^{\circ} \mathrm{C}$ for $30 \mathrm{~s}$, followed by a final extension period of $72{ }^{\circ} \mathrm{C}$ for $5 \mathrm{~min}$. PCR products were analysed on a Pharmacia ALFexpress automated sequencer according to the manufacturer's recommendations.

\section{Statistical analyses}

Genetic variation and estimation of effective population size and migration rate: Exact tests for population differentiation (Raymond and Rousset, 1995a) were performed using the Genepop 3.1 software package (Raymond and Rousset, 1995b). The program FSTAT version 2.9.3 (Goudet, 1995) was used to test for deviations from Hardy-Weinberg proportions and gametic phase disequilibria between pairs of loci, as well as estimating allelic richness (El Mousadik and Petit, 1996), a measure of number of alleles independent of sample size, and expected heterozygosity. In all cases of multiple tests, type I errors were minimized by sequential Bonferroni correction (Rice, 1989).

The spatio-temporal distribution of genetic variance was analysed by a hierarchical analysis of molecular variance in which total genetic variance was partitioned into four hierarchical levels: (i) among regions, (ii) among populations within regions, (iii) among temporal samples within populations and (iv) within samples. These analyses were performed using the HIERFSTAT (Goudet, 2005) R package (R Development Core Team, 2006). To visualize population structure, we performed a
Multidimensional Scaling Analysis based on $\mathrm{F}_{\mathrm{ST}}$ estimates, using the software Vista 5.6.3 (Young, 1996).

Effective population sizes and migration rates for subpopulations within the Odderaa River system were estimated with the software MLNE2 (Wang and Whitlock, 2003) using a likelihood-based implementation of the temporal method. The analyses were based on temporal samples from the focal population, where all other samples from the system were pooled into a 'source pool', representing the allele frequencies of immigrants from within the river system. Although immigration from the populations outside the system is also a possibility, this is assumed to be quantitatively less important than gene flow at the microgeographical scale within the system. For all estimates, a generation length of 3.5 years (Hansen et al., 2002) and an upper limit of 1000 for $N_{\mathrm{e}}$ was assumed. Estimates of effective population sizes and migration rates for the Lake Hald drainage were obtained from Jensen et al. (2005).

Tests for selection: We aimed at investigating the effect of selection by using a number of different tests. We did this for two reasons: (i) as discussed by Storz (2005) empirically and model-based tests each hold both advantages and disadvantages. While empirically based tests accurately capture the demographic history of populations, they heavily rely on the loci under study being a representative sample of the genome. The modelbased tests on the other hand are expected to show less dependence on the loci analysed, but may rely on erroneous assumptions. We therefore applied both types of tests to validate their performance. (ii) By the use of more than one test of selection we aimed at minimizing the amount of type I errors as we only considered rejections of the hypothesis of neutrality as true indications of selection if they were supported by more than one test. Due to the high number of pairwise tests, sequential Bonferroni correction would be overly conservative, when applying the above approach. Significance levels were therefore corrected for multiple comparisons by controlling the false discovery rate following the procedure by Benjamini and Yekutieli (2001).

A direct comparison of genetic differentiation at the presumably neutral microsatellite loci and the TAP2 markers provides an empirically based test of selection. While $\mathrm{F}_{\mathrm{ST}}$ is potentially bound by the level of homozygosity within samples (Charlesworth, 1998; Hedrick, 1999), this is only expected to be of importance when mutation exceeds migration (Hedrick, 2005). The high migration rates observed within these systems suggest that $\mathrm{F}_{\mathrm{ST}}$ is the most appropriate estimator of genetic differentiation compared to a standardized estimator, such as G' $_{\text {ST }}$ (Hedrick, 2005). F FT was estimated using the program FSTAT version 2.9.3 (Goudet, 1995) and associated $99 \%$ confidence intervals were obtained by bootstrapping over loci (15000 permutations). Point estimates for the TAP2 markers lying outside this confidence interval were considered to be significant (Weir, 1996).

To model the neutral expectation of genetic differentiation we applied the method by Beaumont and Nichols (1996) using the program FDIST2. This method builds the expected neutral distribution of $\mathrm{F}_{\mathrm{ST}}$ as a function of heterozygosity, based on coalescent simulations, to which the observed distribution of $\mathrm{F}_{\mathrm{ST}}$ at 
individual loci can be compared. Beaumont and Nichols (1996) showed that this procedure is rather robust to many violations in assumptions used to generate the expected distribution, for example model of population structure and mutation rate, and the approach has been applied to detect selection in several studies (Storz and Dubach, 2004; Vasemägi et al., 2005; Aguilar and Garza, 2006). Specifically, all simulations were based on 50000 realizations assuming a stepwise mutation model. We conducted this test at different hierarchical levels. For the largest geographical scale we considered contemporary samples from all three regions collectively, that is this level consisted of the 1992 samples from Odderaa River, the 1989 samples from Lake Hald and the Gudenaa River samples. The small geographical level was assessed by considering samples from each region separately. Again we only considered samples originating from the same year. Furthermore, we conducted the test for all pairs of populations originating from the same region and year. Finally, we tested for neutrality at the temporal scale by conducting the test for pairs of temporal samples originating from the same locality. When considering all samples we assumed a total number of demes of 100, while within the Odderaa River, Gudenaa River and Lake Hald system we assumed a total number of demes of 20,30 and 10 , respectively.

As noted by Beaumont and Nichols (1996), their method has reduced power particularly when the number of samples is small. We therefore additionally applied the method by Bonhomme et al. (submitted) when considering pairs of populations within regions. Also, we applied this test to pairs of temporal samples within populations. This procedure, which is implemented in the program Neutrallelix (http://www.genetix. univ-montp2.fr/ genetix/neutrality.htm), evaluates the test statistic $\Delta=\theta_{\mathrm{WC}}-\theta_{\mathrm{RH}}$, where $\theta_{\mathrm{WC}}$ is the multiallelic $\mathrm{F}_{\mathrm{ST}}$ estimate based on Weir and Cockerham (1984) and $\theta_{\mathrm{RH}}$ as defined by Robertson and Hill (1984). The observed $\Delta$ is compared to a simulated neutral distribution and neutrality is rejected if the probability of belonging to the simulated distribution is below the significance level. The method takes advantage of the property that the two estimators weight rare and common alleles differently when combining biallelic estimates into the multiallelic estimate. Selection influencing the allele frequency distribution at a locus leads to different average biallelic $\theta_{\mathrm{u}}$ estimates for rare and common alleles, causing $\Delta$ to deviate from neutral expectations. As shown by Bonhomme et al. (submitted), this approach is robust to deviations from the assumed model of population structure and migration-drift equilibrium and has been used to demonstrate selection at two allozyme loci in populations of pearl oyster (Pinctada margaritifera) (Arnaud-Haond et al., 2003). As the method ignores the effect of mutation by assuming that migration exceeds mutation, we only applied it within systems where we did not expect mutation to play a significant role given the high observed migration rates. We simulated, using 1000 iterations, the neutral distribution of $\Delta$ assuming values of effective population size $(N)$ and migration rate $(m)$ based on multilocus $\mathrm{F}_{\mathrm{ST}}$ estimates, as recommended by Bonhomme et al. (submitted). In all simulations we assumed an infinite island model and allowed 100 generations to reach migrationdrift equilibrium. $F_{\mathrm{ST}}$ based on the estimators by Weir and Cockerham (1984) and Robertson and Hill (1984) was calculated using the program Genetix ver. 4.05.2 (Belkhir et al., 2004).

Finally, we tested for signals of selective sweeps, that is evidence of directional selection, by evaluating the LnRH statistic, given as the natural logarithm of the ratio of $(1 /(1-\mathrm{He}))^{2}-1$, where He denotes the expected heterozygosity for each pair of populations (Kauer et al., 2003). A selective sweep is expected to reduce the genetic variance at the genomic region affected by selection, while neutrally evolving regions are left unaffected. Thus the distribution of the ratio of observed genetic variance over loci in two populations provides a mutation rate independent test statistic suitable for detection of past selective sweeps (Schlötterer, 2002). The natural logarithm of this ratio for neutrally evolving loci has been shown to approximate a Gaussian distribution (Schlötterer, 2002; Kauer et al., 2003). We tested the specific hypothesis of a selective sweep at the TAP2 markers, by fitting a Gaussian distribution to each of 1000 bootstrapped sets of neutral LnRH estimates and calculating the probability of the estimate for the TAP2 markers.

\section{Results}

Neutral genetic variability and population structure

No significant deviations from Hardy-Weinberg proportions or gametic phase disequilibria were detected at the presumably neutral markers after correcting for multiple comparisons. Summary statistics are provided in Supplementary Table S1. For the presumably neutral markers an overall $F_{\text {ST }}$ estimate of $0.040(0.029-0.052)$ was obtained, with pairwise estimates ranging from 0.000 to 0.106 , the highest of which were observed among populations from different regions (Supplementary Table S2). The hierarchical analysis of the distribution of analysis of molecular variance provided further insight into the spatio-temporal population structure (Table 2). A significant proportion of the total neutral genetic variance was distributed among regions $(3.7 \%$ of total variance; $95 \%$ confidence interval: $(1.9-5.7 \%) ; P=0.01)$, indicating a temporally stable population structure among regions. This was further supported by the Multidimensional Scaling Analysis plot which reflected the regional geographical structure well, by revealing three distinct clusters representing the three regions (Figure 2). Within regions, a much lower, yet significant, proportion of the genetic variance was distributed among populations ( $0.6 \%$ of total variance, $P=0.01)$, while a larger proportion was distributed among temporal samples within populations $(1.2 \%$ of total variance, $P=0.001$ ). This indicates a temporally unstable population structure within drainages.

Estimates of effective population sizes were low, ranging from 37 to 90 within the Odderaa River system and 37-54 in the Lake Hald system. The joint estimates of migration rates were very high, ranging from 0.41 to 1 within the Odderaa River system and 0.15-0.26 within the Lake Hald system (Table 3).

Population structure and polymorphism at TAP2 markers Summary statistics for the TAP2 markers are provided in Supplementary Table S3. One significant departure from Hardy-Weinberg equilibrium was revealed at the TAP2B 
Table 2 Hierarchical analysis of molecular variance for the three classes of markers

\begin{tabular}{|c|c|c|c|}
\hline Hierarchical level & $\begin{array}{c}\text { Variance } \\
\text { component } \\
(\%)\end{array}$ & d.f. & P-value \\
\hline \multicolumn{4}{|l|}{ Neutral markers } \\
\hline Among regions & 3.7 & 2 & 0.01 \\
\hline Among populations within regions & 0.6 & 9 & 0.01 \\
\hline $\begin{array}{l}\text { Among temporal samples within } \\
\text { populations }\end{array}$ & 1.2 & 9 & 0.001 \\
\hline Within samples & 94.5 & 1869 & $<0.001$ \\
\hline \multicolumn{4}{|l|}{ TAP2A marker } \\
\hline Among regions & 2.4 & 2 & 0.01 \\
\hline Among populations within regions & 0.4 & 9 & 0.01 \\
\hline $\begin{array}{l}\text { Among temporal samples within } \\
\text { populations }\end{array}$ & 1.9 & 9 & 0.01 \\
\hline Within samples & 95.3 & 1869 & $<0.001$ \\
\hline \multicolumn{4}{|l|}{ TAP2B marker } \\
\hline Among regions & 2.7 & 2 & 0.01 \\
\hline Among populations within regions & 1.6 & 9 & 0.01 \\
\hline $\begin{array}{l}\text { Among temporal samples within } \\
\text { populations }\end{array}$ & 0.5 & 9 & 0.01 \\
\hline Within samples & 95.2 & 1869 & $<0.001$ \\
\hline
\end{tabular}

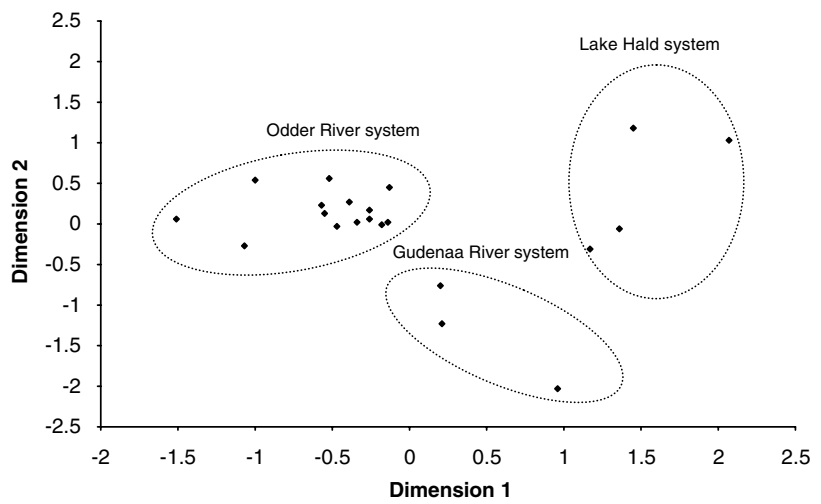

Figure 2 Multi Dimensional Scaling Analysis plot based on $\mathrm{F}_{\mathrm{ST}}$ estimates (Weir and Cockerham, 1984) for eight neutral microsatellite loci. Dimension 1 explains $38 \%$ of the total variance, while dimension 2 explains $22 \%$.

Table 3 Joint estimates of effective population sizes, $N_{\mathrm{e}}$ migration rates, $m$, and corresponding 95\% confidence intervals as obtained by the likelihood-based temporal method of Wang and Whitlock (2003)

\begin{tabular}{lccccc}
\hline Population & $T$ & $\mathrm{~N}_{e}$ & $95 \% \mathrm{CI}$ & $\mathrm{m}$ & $95 \% \mathrm{CI}$ \\
\hline ODR2 & 3.4 & 83 & $29-536$ & 0.53 & $0.27-1.00$ \\
ODR1 & 3.4 & 48 & $27-99$ & 0.46 & $0.24-1.00$ \\
KRAG & 3.4 & 38 & $23-96$ & 1.00 & $0.36-1.00$ \\
FISK & 3.4 & 37 & $22-79$ & 0.72 & $0.31-1.00$ \\
STAM & 3.4 & 68 & $27-298$ & 0.55 & $0.25-1.00$ \\
ASSE & 3.4 & 90 & $28-570$ & 0.41 & $0.20-1.00$ \\
ASBK & 3.4 & 56 & $29-214$ & 1.00 & $0.40-1.00$ \\
KRO & 3.71 & 37 & $25-58$ & 0.15 & $0.07-0.25$ \\
KAP & 3.71 & 54 & $28-140$ & 0.26 & $0.15-0.42$ \\
\hline
\end{tabular}

Abbreviation: $\mathrm{CI}$, confidence interval.

$\mathrm{T}$ denotes number of generations between temporal samples. Estimates for the samples $\mathrm{KRO}$ and KAP are reproduced from Jensen et al. (2005). marker in the KRO89 sample (Supplementary Table S3). No linkage disequilibrium was observed between the $T A P 2 A$ and TAP $2 B$ markers, concordant with the mapping of these loci to two different linkage groups in Atlantic salmon (Grimholt et al., 2002). Also, no linkage disequilibrium was observed between any of the TAP2 markers and presumably neutral markers. Overall, levels of population differentiation similar to that found for the presumably neutral markers were observed at the TAP2 markers $\left(T A P 2 A: \mathrm{F}_{\mathrm{ST}}=0.038\right.$; TAP2B: $\left.\mathrm{F}_{\mathrm{ST}}=0.037\right)$. However, pairwise $\mathrm{F}_{\mathrm{ST}}$ estimates for the TAP2A (ranging from 0.000 to 0.178 ) and TAP2B (ranging from 0.000 to 0.356 ) markers seemed to cover a wider interval of values compared to the presumably neutral markers (Supplementary Table S4).

\section{Temporal population structure at TAP2 markers}

When comparing $\mathrm{F}_{\mathrm{ST}}$ estimates for TAP2A and the neutral loci among pairs of temporal samples from the same locality three outliers at the TAP2A marker (out of nine comparisons) were revealed, two of which were high outliers (Figure 3 and Supplementary Table S4). In contrast, only one significantly high $\mathrm{F}_{\mathrm{ST}}$ outlier was observed at the TAP2B marker (Figure 3 and Supplementary Table S4).

When applying the test by Beaumont and Nichols (1996) to temporal samples within populations, the TAP2A marker was identified as a high outlier in KAP after correcting for multiple comparisons $(P=0.010$, critical $P$-value $=0.018)$. No other loci were indicated at this level. The hypothesis of neutrality within KAP was also rejected by the Neutrallelix test. One additional locality, that is ODR1, was marginally significant (Table 4). KAP was also identified as a high outlier in the $\mathrm{F}_{\mathrm{ST}}$ comparison, while ODR1 was significant at the 95\% significance level. For the TAP2B marker, the Neutrallelix test did not reject the neutral null hypothesis of neutrality in any case for the temporal samples. The congruent results for TAP2A suggest that the significant cases for KAP are not merely type I errors and that selection might fluctuate within this locality over the time period covered in this study. The same might apply for ODR1. In further support of natural selection in KAP, a selective sweep over time was suggested at the TAP2A marker within this sample using the method by Schlötterer (2002) and Kauer et al. (2003). In addition to this, a marginally significant selective sweep was indicated in the ODR1 and ODR2 sample (Figure 4).

\section{Geographical population structure at TAP2 markers}

At the largest geographical scale, encompassing all populations from all three regions, the neutrality test by Beaumont and Nichols (1996) did not suggest any of the TAP2-linked markers deviate from neutral expectations, but identified locus Ssa85 as a high outlier $(P=0.013)$ at this scale. In contrast to these results, the comparison of pairwise $\mathrm{F}_{\mathrm{ST}}$ estimates among contemporary samples from different regions identified 17 and 27 outliers at the TAP2A and TAP $2 B$ markers, respectively. While the TAP2A marker showed high outliers at the highest proportion, the TAP2B marker was mainly dominated by low outliers (Figure3 and Supplementary Table S4). As the assumption of no mutation is likely to 

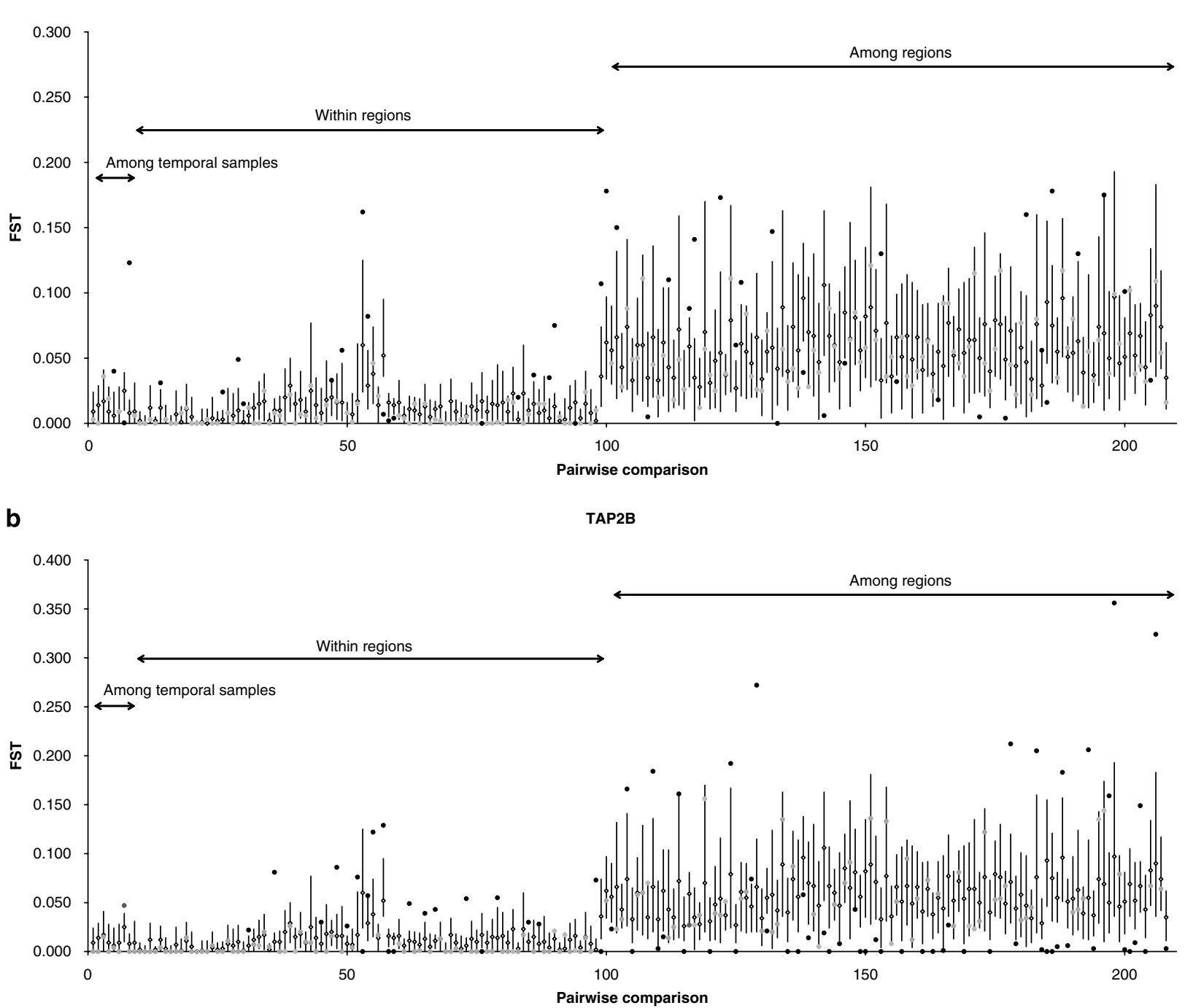

Figure 3 Pairwise comparisons of genetic differentiation as determined by $\mathrm{F}_{\mathrm{ST}}$ for neutral and TAP-linked microsatellite loci. Estimates for the TAP-linked markers are contrasted to the $99 \%$ confidence intervals for the neutral loci, represented by vertical bars. $\diamond$, point estimate of $\mathrm{F}_{\mathrm{ST}}$ for neutral microsatellite loci; $\bigcirc$, point estimate of $\mathrm{F}_{\mathrm{ST}}$ for $T A P$-linked marker with $P>0.01 ; \bullet$, point estimate of $\mathrm{F}_{\mathrm{ST}}$ for $T A P$-linked marker with $P<0.01$. (a) TAP2A-linked marker, (b) TAP2B-linked marker

be violated when comparing samples among regions, we did not apply the Neutrallelix test.

At the within-region scale the test by Beaumont and Nichols (1996) identified none of the TAP2-linked markers as outliers. However, locus T3-13 was identified as an outlier within the 1992 samples from Odderaa River $(P=0.014$, critical $P$-value $=0.022)$. When conducting the test for pairs of populations from within the same regions and sampled the same year, no loci were identified as outliers. However, T3-13 was a marginally significant outlier in two pairwise tests in accord with this locus being identified as an outlier when considering the 1992 samples from Odderaa River collectively.

When comparing pairwise $\mathrm{F}_{\mathrm{ST}}$ estimates between contemporary samples within systems, 14 cases of outliers were observed for the TAP $2 A$ marker and 13 outliers for $T A P 2 B$ (Figure 3 and Supplementary Table S4). Applying the Neutrallelix test revealed a total of three significant deviations from neutral expectations at the TAP $2 A$ marker and an additional three significant deviations at the $T A P 2 B$ marker after correcting for multiple comparisons (Table 4). In four out of those six tests that were significant in the Neutrallelix test, the same pair of samples was involved in a significant outcome in the $\mathrm{F}_{\mathrm{ST}}$ comparison.

The test for selective sweeps by Schlötterer (2002) and Kauer et al. (2003) revealed a significantly reduced variability at the TAP $2 A$ marker in 20 pairwise tests after correcting for multiple comparisons, 18 of these involving KAP89 and 2 involving ODR2-92 (Figure 4). In addition, four tests were marginally significant, three of those involving ODR1-04. For ODR2-92 and ODR1-04, $T A P 2 A$ was also indicated to be subject to selection using the $\mathrm{F}_{\mathrm{ST}}$ and the Neutrallelix approaches. At the TAP2B marker no indications of selective sweeps were evident.

\section{Discussion}

In this study, we analysed genetic variation at markers linked to two non-MHC immune genes, that is, TAP2A 
Table 4 Estimates of $\Delta$ given as $\theta_{\mathrm{WC}}-\theta_{\mathrm{RH}}$ along with corresponding $P$-values from the Neutrallelix test of Bonhomme et al. (submitted) among localities within river systems (a) and among temporal samples within localities (b)

\begin{tabular}{|c|c|c|c|c|}
\hline & \multicolumn{2}{|c|}{ TAP2A } & \multicolumn{2}{|c|}{ TAP2B } \\
\hline & $\Delta$ & P-value & $\Delta$ & P-value \\
\hline \multicolumn{5}{|l|}{ (a) } \\
\hline \multicolumn{5}{|l|}{ Populations } \\
\hline \multicolumn{5}{|l|}{ River Odderaa } \\
\hline ASBK92-FISK92 & 0.00024 & 0.431 & -0.00001 & 0.531 \\
\hline ASBK92-KRAG92 & 0.00057 & 0.395 & -0.00017 & 0.619 \\
\hline ASBK92-ODR1-92 & -0.00362 & 0.852 & 0.00010 & 0.413 \\
\hline ASBK92-ODR2-92 & -0.00382 & 0.841 & -0.00040 & 0.735 \\
\hline ASBK92-ASSE92 & 0.00991 & 0.033 & -0.00148 & 0.826 \\
\hline ASBK92-STAM92 & -0.00488 & 0.987 & 0.00018 & 0.417 \\
\hline FISK92-KRAG92 & -0.00365 & 0.873 & -0.00015 & 0.602 \\
\hline FISK92-ODR1-92 & -0.00234 & 0.768 & -0.00009 & 0.583 \\
\hline FISK92-ODR2-92 & 0.00177 & 0.310 & 0.00023 & 0.351 \\
\hline FISK92-ASSE92 & 0.00071 & 0.363 & 0.00055 & 0.319 \\
\hline FISK92-STAM92 & -0.00185 & 0.884 & -0.00059 & 0.730 \\
\hline KRAG92-ODR1-92 & -0.00459 & 0.898 & 0.00033 & 0.309 \\
\hline KRAG92-ODR2-92 & 0.00292 & 0.204 & -0.00031 & 0.737 \\
\hline KRAG92-ASSE92 & -0.00001 & 0.480 & -0.00196 & 0.864 \\
\hline KRAG92-STAM92 & -0.00384 & 0.965 & 0.00047 & 0.323 \\
\hline ODR1-92-ODR2-92 & 0.00820 & 0.048 & -0.00004 & 0.549 \\
\hline ODR1-92-ASSE92 & 0.00019 & 0.436 & -0.00105 & 0.773 \\
\hline ODR1-92-STAM92 & -0.00739 & $0.996^{*}$ & 0.00018 & 0.416 \\
\hline ODR2-92-ASSE92 & 0.02636 & $0.002^{*}$ & -0.00048 & 0.664 \\
\hline ODR2-92-STAM92 & 0.00039 & 0.411 & -0.00029 & 0.653 \\
\hline ASSE92-STAM92 & 0.00374 & 0.051 & -0.00124 & 0.857 \\
\hline ASBK04-FISK04 & -0.00331 & 0.841 & -0.00080 & 0.727 \\
\hline ASBK04-KRAG04 & -0.00574 & 0.946 & 0.00098 & 0.297 \\
\hline ASBK04-ODR1-04 & 0.01227 & 0.033 & 0.00225 & 0.165 \\
\hline ASBK04-ODR2-04 & -0.00023 & 0.541 & 0.00002 & 0.498 \\
\hline ASBK04-ASSE04 & 0.00031 & 0.486 & -0.01265 & $0.998^{*}$ \\
\hline ASBK04-STAM04 & -0.00020 & 0.564 & 0.00057 & 0.386 \\
\hline FISK04-KRAG04 & -0.00363 & 0.831 & 0.00016 & 0.459 \\
\hline FISK04-ODR1-04 & 0.01577 & 0.063 & -0.00367 & 0.803 \\
\hline FISK04-ODR2-04 & -0.00151 & 0.722 & 0.00004 & 0.507 \\
\hline FISK04-ASSE04 & 0.00272 & 0.286 & -0.01110 & $0.996^{*}$ \\
\hline FISK04-STAM04 & 0.00374 & 0.230 & -0.00307 & 0.820 \\
\hline KRAG04-ODR1-04 & 0.01538 & 0.055 & -0.00395 & 0.809 \\
\hline KRAG04-ODR2-04 & -0.00335 & 0.862 & -0.00002 & 0.530 \\
\hline KRAG04-ASSE04 & -0.00502 & 0.881 & -0.01258 & 0.938 \\
\hline KRAG04-STAM04 & 0.00038 & 0.483 & -0.00211 & 0.773 \\
\hline ODR1-04-ODR2-04 & 0.01758 & 0.042 & -0.00422 & 0.827 \\
\hline ODR1-04-ASSE04 & 0.01263 & 0.062 & -0.01718 & 0.983 \\
\hline ODR1-04-STAM04 & 0.03634 & $0.001^{*}$ & 0.00030 & 0.429 \\
\hline ODR2-04-ASSE04 & -0.00162 & 0.773 & -0.01238 & $0.998^{*}$ \\
\hline ODR2-04-STAM04 & -0.00280 & 0.758 & -0.00280 & 0.806 \\
\hline ASSE04-STAM04 & 0.01023 & 0.064 & -0.01655 & 0.991 \\
\hline \multicolumn{5}{|l|}{ Lake Hald } \\
\hline KRO89-KAP89 & 0.09906 & 0.012 & -0.00342 & 0.664 \\
\hline KRO02-KAP02 & 0.02290 & 0.116 & 0.03575 & 0.090 \\
\hline \multicolumn{5}{|l|}{ Gudenaa River } \\
\hline TN94-TO94 & 0.01486 & 0.203 & 0.07577 & 0.019 \\
\hline TN94-BR95 & 0.00693 & 0.415 & -0.00568 & 0.853 \\
\hline TO94-BR95 & -0.00122 & 0.673 & 0.00843 & 0.126 \\
\hline \multicolumn{5}{|l|}{ (b) } \\
\hline \multicolumn{5}{|l|}{ Locality } \\
\hline ASBK & 0.00182 & 0.266 & 0.00047 & 0.313 \\
\hline FISK & -0.00220 & 0.736 & 0.00010 & 0.436 \\
\hline KRAG & -0.00702 & 0.966 & -0.00007 & 0.526 \\
\hline ODR1 & 0.02515 & 0.009 & -0.00073 & 0.652 \\
\hline ODR2 & 0.00645 & 0.058 & 0.00002 & 0.541 \\
\hline ASSE & 0.01567 & 0.018 & -0.00732 & 0.974 \\
\hline STAM & -0.00384 & 0.969 & 0.00030 & 0.380 \\
\hline $\mathrm{KRO}$ & -0.00098 & 0.640 & 0.01932 & 0.092 \\
\hline KAP & 0.07543 & $0.004^{*}$ & -0.00367 & 0.701 \\
\hline
\end{tabular}

\footnotetext{
* Indicates significant departure from neutrality at the 5\% significance level after correcting for multiple comparisons by controlling the false discovery rate following Benjamini and Yekutieli (2001). All simulations, based on 1000 iterations, were performed assuming an infinite island model of migration and using estimates of $N_{\mathrm{e}}$ and $m$ obtained from $\mathrm{F}_{\mathrm{ST}}$ estimates as recommend by Bonhomme et al. (submitted).
} 


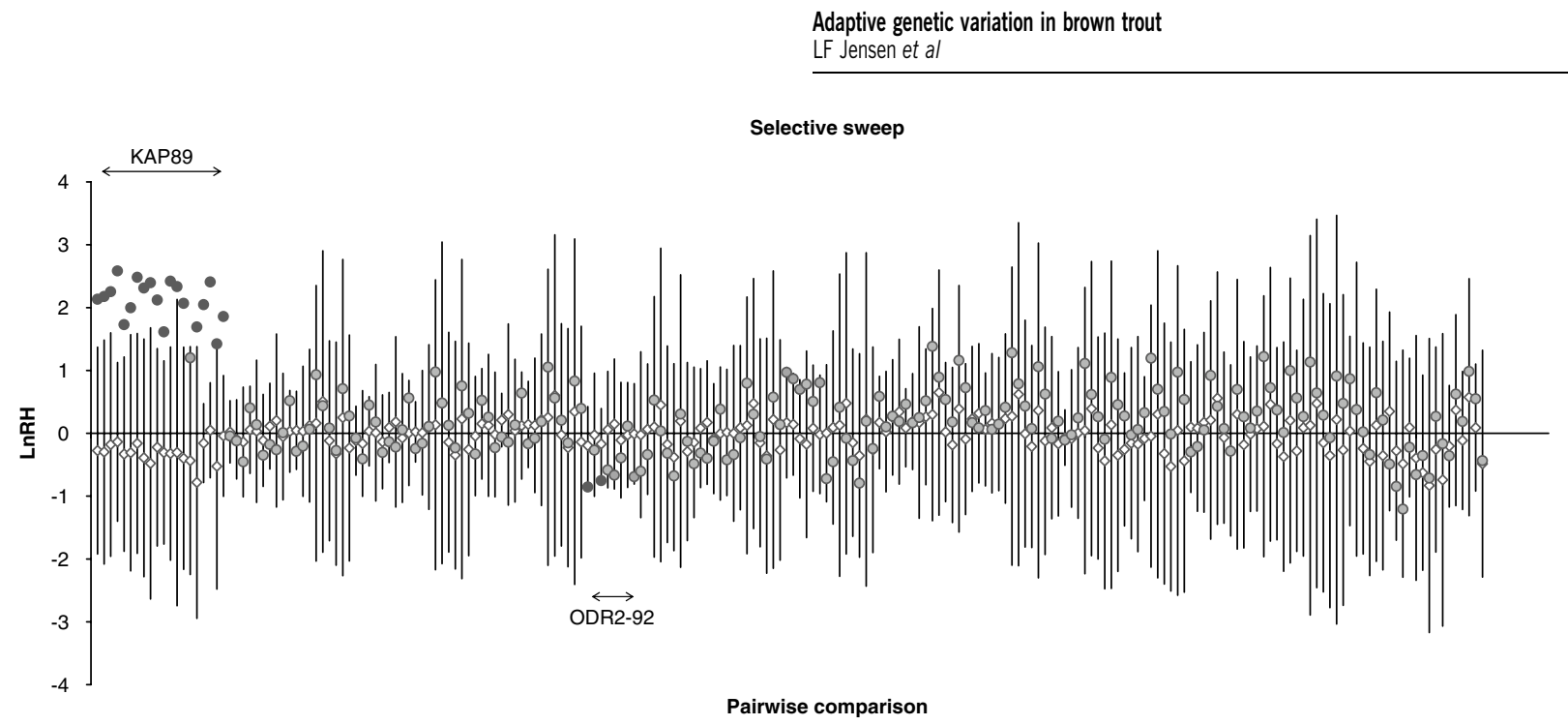

Figure 4 Test for selective sweeps (Schlötterer, 2002) by evaluation of the LnRH test statistic as defined by Kauer et al. (2003). Vertical bars denote the $99 \%$ confidence interval for neutral microsatellite loci to which LnRH for the TAP2A marker is contrasted. $\diamond$, mean point estimate of LnRH for neutral microsatellite loci; $\bigcirc$, point estimate of LnRH for the TAP2A marker with $P>0.1, \boldsymbol{\Delta} 0.05>P<0.01, \bullet P<0.01$.

and $T A P 2 B$ together with eight presumably neutral microsatellite loci. The analyses provided some indications of divergent selection at both temporal and spatial scale. Before we turn to a discussion of these issues, the special pattern observed at the TAP2B marker calls for some consideration. The TAP $2 B$ marker showed considerably less variability than the TAP $2 A$ marker, both with respect to total number of alleles and expected heterozygosity (see Supplementary Table S3). If the mutation rate at the TAP2B marker locus is much lower than at the functional locus, each marker allele could be linked to several functional alleles at the $T A P 2 B$ locus. Such a pattern was found for a marker linked to the $U B A$ locus in Atlantic salmon (Salmo salar) by Grimholt et al. (2002). If this is also the case in brown trout, this linkage pattern could result in generally lowered population differentiation at the marker locus as observed in this study, even in the case of weak directional selection at the underlying functional locus. However, inflated population differentiation at the marker locus is not expected to result from this linkage pattern. Due to this possible bias, in the following, we only consider high $\mathrm{F}_{\mathrm{ST}}$ outliers at the TAP2B marker.

\section{Evidence of temporally varying selection}

In this study we found some indications of temporally fluctuating selection at the TAP2A marker. First of all, the test by Beaumont and Nichols (1996) indicated that TAP2A was subject to selection over time in KAP. The comparison of $\mathrm{F}_{\mathrm{ST}}$ estimates, the Neutrallelix test and the test for selective sweeps agreed with this by identifying KAP as subject to temporally varying selection. The similar results produced by the different selection tests add confidence to the notion that the significant outcomes actually reflect temporally varying selection and are not merely due to chance. Especially, the congruent results from the Neutrallelix test (Bonhomme et al., submitted) points to this, since this test and the $\mathrm{F}_{\mathrm{ST}}$ outlier tests $\left(\mathrm{F}_{\mathrm{ST}}\right.$ comparison and the test by Beaumont and Nichols, 1996) make use of different information. While the latter evaluates measures of population differentiation per se, the Neutrallelix test measures the statistic $\Delta$, the difference among estimators of genetic differentiation, and is thus based on a completely different approach. The sample originating from KAP $(n=22)$ was the smallest sample analysed in this study. However, we do not consider it likely that the small sample size has severely affected the results from the selection tests. Thus, Beaumont and Nichols (1996) found that samples of 25 diploid individuals are sufficient when applying their FDIST2 test and that smaller sample sizes rather made the test more conservative. Similar sample sizes have also been found to produce low coefficients of variance for estimates of $\mathrm{F}_{\mathrm{ST}}$ at highly variable loci (Kalinowski, 2005). Could the observed pattern be due to demographic events such as population bottlenecks, which potentially increase the variance in, for example, $F_{S T}$ among loci, rather than natural selection? Jensen et al. (2005) did in fact find some signals of population bottlenecks within the samples from KAP but the signal was not particularly strong. To further assess whether population bottlenecks could have generated the observed patterns we applied the bottleneck test by Cornuet and Luikart (1996), assuming a twophase model of mutation with $10 \%$ non-stepwise mutations and the test by Garza and Williamson (2001) assuming a two-phase model of mutation with $10 \%$ nonstepwise mutations, mean size of non one-step mutations of 3.5 repeat units and $\theta$ of 0.4 . The bottleneck test by Cornuet and Luikart (1996) only indicated a bottleneck in the STAM04 sample, while the test by Garza and Williamson (2001) suggested a bottleneck in the KRO89 sample after correcting for multiple comparisons using the procedure by Benjamini and Yekutieli (2001) (data not shown). Given that the indications of population bottlenecks are either weak or missing we do not consider it likely that the increased $\mathrm{F}_{\mathrm{ST}}$ estimates at the TAP2A marker and the congruent significant Neutrallelix tests are a result of population bottlenecks, but rather suggest that they result from divergent natural selection.

Temporally varying selection within localities was further indicated by the finding of a selective sweep when comparing the temporal samples from KAP. The 
selective sweep seems to have appeared in the KAP89 sample. However, we did not find any footprints of this selective sweep in the KAP02 sample. How could the signal disappear over a period of 13 years? The study by Jensen et al. (2005) found migration rate among the populations within the Lake Hald system to be high, ranging from 0.13 to 0.36 . It is therefore possible that the signal of a selective sweep in KAP89 could have been 'swamped' by extensive immigration in the following 13 years. Though not significantly deviating from HardyWeinberg expectations, we did observe fewer heterozygotes than expected in both the KAP89 and the KAP02 sample and we cannot rule out that this might also have influenced the test results.

Given the central role of TAP molecules in the adaptive immune system, the indications of temporally fluctuating natural selection could be due to fluctuating compositions of the parasite and pathogen fauna. Such fluctuations in pathogen distributions have been suggested in populations of salmonids (Roberts, 1993), and a few studies of $M H C$ variation have found temporally fluctuating selection at these genes as well (Westerdahl et al., 2004; Charbonnel and Pemberton, 2005; Coughlan et al., 2006). However, Miller et al. (2001) and Hansen et al. (2007) did not find any trend of temporally varying allele frequencies at $M H C$ genes in populations of sockeye salmon (Oncorhynchus nerka) and brown trout respectively. While studies now suggest that temporally fluctuating selection might be just as important to evolutionary responses in wild populations as spatial heterogeneities, this issue needs to be further addressed to investigate the generality of this finding as well as the width of the time intervals over which these fluctuations occur.

\section{Geographical patterns of selection}

In this study we also found some indications of selection at a spatial scale, although they were not as convincing as for the temporal scale. The indication of selection seemed to be most pronounced at small geographical scales. Thus, at this scale the pairwise comparisons of $\mathrm{F}_{\mathrm{ST}}$ estimates and the Neutrallelix test produced congruent results in several cases. Furthermore, a number of selective sweeps were indicated among samples from the same region and year. Indications of divergent selection across a very small geographical scale have also been reported for the $M H C$ class II locus in Atlantic salmon (Landry and Bernatchez, 2001). These authors suggested that heterogeneously distributed parasites or pathogens at this small scale were responsible. Such diverse patterns of parasite distributions in fish populations have been proposed by Bakke and Harris (1998). This suggests that salmonids might experience highly heterogeneous selection regimes at micro-geographical scales with respect to parasites and pathogens, and that local directional selection could be an important evolutionary force at genes engaged in the adaptive immune system. It should be noted that the indications of mainly directional selection at the small geographical scale could be due to low power in detecting balancing selection due to generally weak population differentiation at this scale. While low levels of population differentiation at small geographical scales provide power to detect divergent selection, at the same time power to detect balancing selection at this scale is low. The opposite can apply at the large geographical scale if population differentiation is high. We ascribe the identification of T3-13 as an outlier within the Odderaa River to chance.

The limited evidence of divergent natural selection at the large geographical scale suggests that no stable local adaptations at TAP polymorphism exist at the regional level. A similar finding was reported by Campos et al. (2006) for MHC class II polymorphism among Spanish brown trout populations. Local adaptation to different parasite and pathogen faunas, if present, might be expected to be found at larger geographical scales than those covered by these studies. This is in agreement with Hansen et al. (2002), who predicted that local adaptations are most likely to be found at the level of several river systems rather than at the scale of individual rivers unless selection is very strong. The demonstration of differences in susceptibility to specific parasites among wild populations of salmonids (Bakke et al., 1990, 2004; Dalgaard et al., 2003) as well as other species of teleost fishes (Gleeson et al., 2000; Kalbe and Kurtz, 2006) suggests that local parasite and pathogen faunas exert a significant selective pressure on wild populations and that local adaptation to parasite faunas might be expected at the larger geographical scale. Indeed, Hansen et al. (2007) found temporally stable and possibly adaptive divergence at a $M H C$ class I-linked microsatellite locus between two Norwegian brown trout populations suggesting that these populations are locally adapted at $\mathrm{MHC}$ loci.

In the present study, locus Ssa85 was also identified as an outlier at a large geographical scale, but not within systems. Interestingly, Aguilar and Garza (2006) also identified locus Ssa85 as an outlier at the highest geographical level when analysing genetic variation in populations of California coastal steelhead trout. The finding of this marker being associated with thermal tolerance (Danzmann et al., 1999) and spawning time (Sakamoto et al., 1999) in rainbow trout suggests that adaptations in these traits might occur within Danish brown trout populations as well as in California coastal steelhead trout.

\section{A role for non- $M H C$ variation in adaptive divergence}

In this study, we set out to investigate whether immune genes other than classical MHC class I and II loci might be subject to selection in natural populations. Traditionally, most studies addressing adaptive evolution of immune genes have focused on MHC genes. Clearly, for these genes the most compelling evidence of natural selection has been gathered, which, along with detailed knowledge about structure and function of the encoded molecules as well as selective agents, makes these genes obvious candidates for such studies. However, studies that have quantified the contribution from $M H C$ polymorphism to the variability in immune response often find this contribution to be rather small, accounting only for a few percent (Jepson et al., 1997). Given the complexity of the adaptive immune system this might not be entirely unexpected. However, natural selection at non-MHC immune genes is rarely addressed, even though such genes might be of importance to fitness. The genes encoding the TAP molecules could be of importance to the efficiency of the immune response and 
thus of importance to fitness. The results from this study indicate that this might indeed be the case, at least in brown trout. Thus, our results suggest that more attention to non-MHC immune genes is warranted, as this could lead to further insight into adaptive evolution in wild populations and the evolutionary dynamics of the immune system

\section{Acknowledgements}

We thank Peter Foged Larsen and Erik Madsen for assistance during sampling in the field. LFJ was supported by funding from the International School of Biodiversity Sciences (ISOBIS) and MMH by the Danish Natural Science Research Council (grant no. 272-050202).

\section{References}

Abele R, Tampé R (2006). Modulation of the antigen transport machinery TAP by friends and enemies. FEBS Lett 580: 1156-1163.

Aguilar A, Garza JC (2006). A comparison of variability and population structure for major histocompatibility complex and microsatellite loci in California coastal steelhead (Oncorhynchus mykiss Walbaum). Mol Ecol 15: 923-937.

Arnaud-Haond S, Bonhomme F, Blanc F (2003). Large discrepancies in differentiation of allozymes, nuclear and mitochondrial DNA loci in recently founded Pacific populations of the pearl oyster Pinctada margaritifera. J Evol Biol 16: 388-398.

Bakke TA, Harris PD (1998). Diseases and parasites in wild Atlantic salmon (Salmo salar) populations. Can J Fish Aquat Sci 55: 247-266.

Bakke TA, Harris PD, Hansen H, Cable J, Hansen LP (2004). Susceptibility of Baltic and east Atlantic salmon Salmo salar stocks to Gyrodactylus salaris (Monogenea). Dis Aquat Org 58: 171-177.

Bakke TA, Jansen PA, Hansen LP (1990). Differences in the hostresistance of atlantic salmon, Salmo-salar L, stocks to the monogenean Gyrodactylus-salaris Malmberg, 1957. J Fish Biol 37: 577-587.

Beaumont M, Nichols R (1996). Evaluating loci for use in the genetic analysis of population structure. Proc $R$ Soc Lond $B$ Biol Sci 263: 1619-1626.

Belkhir K, Borsa P, Chikhi P, Raufaste N, Bonhomme F (2004). GENETIX 4.05.2, logiciel sous Windows TM pour la génétique des populations. Laboratoire Génome, Populations, Interactions CNRS UMR 5000, Université de Montpellier II, Montpellier, France.

Benjamini Y, Yekutieli D (2001). The control of false discovery rate in multiple testing under dependency. Ann Stat 29: 11651188 .

Bernatchez L, Landry C (2003). MHC studies in nonmodel vertebrates: what have we learned about natural selection in 15 years? I Evol Biol 16: 363-377.

Black FL, Salzano FM (1981). Evidence for heterosis in the HLA system. Am J Hum Genet 33: 894-899.

Bodmer WF (1972). Evolutionary significance of the HL-A system. Nature 237: 139-145.

Bonhomme F, Belkhir K, Raufaste N: Single locus, multiallelic test of neutrality with two multiallelic estimators of Fst. Mol Ecol (submitted).

Borghans JAM, Beltman JB, De Boer RJ (2004). MHC polymorphism under host-pathogen coevolution. Immunogenetics 55: 732-739.

Campos JL, Posada D, Moran P (2006). Genetic variation at MHC, mitochondrial and microsatellite loci in isolated populations of Brown trout (Salmo trutta). Conserv Genet 7: 515-530.

Charbonnel N, Pemberton J (2005). A long-term genetic survey of an ungulate population reveals balancing selection acting on MHC through spatial and temporal fluctuations in selection. Heredity 95: 377-388.

Charlesworth B (1998). Measures of divergence between populations and the effect of forces that reduce variability. Mol Biol Evol 15: 538-543.

Cornuet JM, Luikart G (1996). Description and power analysis of two tests for detecting recent population bottlenecks from allele frequency data. Genetics 144: 2001-2014.

Coughlan J, McGinnity P, O'Farrell B, Dillane E, Diserud O, Eyto Ed et al. (2006). Temporal variation in an immune response gene (MHC I) in anadromous Salmo trutta in an Irish river before and during aquaculture activities. ICES J Mar Sci 63: 1248-1255.

Dalgaard MB, Nielsen CV, Buchmann K (2003). Comparative susceptibility of two races of Salmo salar (Baltic Lule river and Atlantic Conon river strains) to infection with Gyrodactylus salaris. Dis Aquat Org 53: 173-176.

Danzmann RG, Jackson TR, Ferguson MM (1999). Epistasis in allelic expression at upper temperature tolerance QTL in rainbow trout. Aquaculture 173: 45-58.

De Boer RJ, Borghans JAM, van Boven M, Kesmir C, Weissing FJ (2004). Heterozygote advantage fails to explain the high degree of polymorphism of the MHC. Immunogenetics 55: 725.

Doherty PC, Zinkernagel RM (1975). Enhanced immunological surveillance in mice heterozygous at $\mathrm{H}-2$ gene complex. Nature 256: 50-52.

El Mousadik A, Petit RJ (1996). High level of genetic differentiation for allelic richness among populations of the argan tree [Argania spinosa (L.) Skeels] endemic to Morocco. Theor Appl Genet 92: 832-839.

Estoup A, Largiader CR, Perrot E, Chourrout D (1996). Rapid one-tube DNA extraction for reliable PCR detection of fish polymorphic markers and transgenes. Mol Mar Bio Biotech 5 : 295-298.

Estoup A, Presa P, Krieg F, Vaiman D, Guyomard R (1993). $(\mathrm{CT})_{\mathrm{n}}$ and $(\mathrm{GT})_{\mathrm{n}}$ microsatellites: a new class of genetic markers for Salmo trutta L. brown trout). Heredity 71: 488-496.

Estoup A, Rousset F, Michalakis Y, Cornuet J-M, Adriamanga M, Guyomard R (1998). Comparative analysis of microsatellite and allozyme markers: a case study investigating microgeographic differentiation in brown trout (Salmo trutta). Mol Ecol 7: 339-353.

Ferguson A (1989). Genetic differences among brown trout (Salmo trutta) stocks and their importance for the conservation and management of the species. Freshw Biol 21: 35-46.

Garza JC, Williamson EG (2001). Detection of reduction in population size using data from microsatellite loci. Mol Ecol 10: 305-318.

Gleeson DJ, McCallum HI, Owens IPF (2000). Differences in initial and acquired resistance to Ichthyohthirius multifiliis between populations of rainbowfish. J Fish Biol 57: 466-475.

Gomez LM, Camargo JF, Castiblanco J, Ruiz-Narváez EA Cadena J, Anaya JM (2006). Analysis of IL1B, TAP1, TAP2 and IKBL polymorphisms on susceptibility to tuberculosis. Tissue Antigens 67: 290-296.

Goudet J (1995). FSTAT (version 1.2) a computer program to calculate F-stastistics. J Hered 86: 485-486.

Goudet J (2005). HIERFSTAT, a package for R to compute and test hierarchical F-statistics. Mol Ecol Notes 5: 184-186.

Grimholt U, Drabløs F, Jørgensen SM, Høyheim B, Stet RJM (2002). The major histocompatibility class I locus in Atlantic salmon (Salmo salar L.): polymorphism, linkage analysis and protein modelling. Immunogenetics 54: 570-581.

Hansen MM (2002). Estimating the long-term effects of stocking domesticated trout into wild brown trout (Salmo trutta) populations: an approach using microsatellite DNA analysis 
of historical and contemporary samples. Mol Ecol 11: 1003-1015.

Hansen MM, Ruzzante DE, Nielsen EE, Bekkevold D, Mensberg K-LD (2002). Long-term effective population sizes, temporal stability of genetic composition and potential for local adaptation in anadromous brown trout (Salmo trutta) populations. Mol Ecol 11: 2523-2535.

Hansen MM, Skaala Ø, Jensen LF, Bekkevold D, Mensberg K-LD (2007). Gene flow, effective population size and selection at major histocompatibility complex genes: brown trout in the Hardanger Fjord, Norway. Mol Ecol 16: 1413-1425.

Hedrick P (2002). Pathogen resistance and genetic variation at MHC loci. Evolution 56: 1902-1908.

Hedrick PW (1999). Perspective: highly variable loci and their interpretation in evolution and conservation. Evolution 53: 313.

Hedrick PW (2005). A standardized genetic differentiation measure. Evolution 59: 1633-1638.

Hedrick PW, Thomson G, Klitz WG (1987). Evolutionary genetics and HLA: another classic example. Biol J Linn Soc 31: 311-331.

Heemels M-T, Ploegh HL (1994). Substrate specificity of allelic variants of the TAP peptide transporter. Immunity $\mathbf{1}$ : 775-784.

Hughes AL, Nei M (1992). Models of host-parasite interaction and MHC polymorphism. Genetics 132: 863-864.

Jensen LF, Hansen MM, Carlsson J, Loeschcke V, Mensberg KLD (2005). Spatial and temporal genetic differentiation and effective population size of brown trout (Salmo trutta, L.) in small Danish rivers. Conserv Genet 6: 615-621.

Jepson A, Banya W, Sisay-Joof F, Hassan-King M, Nunes C, Bennett $S$ et al. (1997). Quantification of the relative contribution of major histocompatibility complex (MHC) and non-MHC genes in human immune responses. Infect Immun 65: 872-876.

Kalbe M, Kurtz J (2006). Local differences in immunocompetence reflect resistance of sticklebacks against the eye fluke Diplostomum pseudospathaceum. Parasitology 132: 105-116.

Kalinowski ST (2005). Do polymorphic loci require large sample sizes to estimate genetic distances? Heredity 94: 33-36.

Kauer MO, Dieringer D, Schlötterer C (2003). A microsatellite variability screen for positive selection associated with the 'out of Africa' habitat expansion of Drosophila melanogaster. Genetics 165: 1137-1148.

Kuzushita N, Hayashi N, Kanto T, Takehara T, Tatsumi T, Katayama $\mathrm{K}$ et al. (1999). Involvement of transporter associated with antigen processing 2 (TAP2) gene polymorphisms in Hepatitis C virus infection. Gastroenterology 116: $1149-1154$

Laikre L, Jarvi T, Johansson L, Palm S, Rubin JF, Glimsater CE et al. (2002). Spatial and temporal population structure of sea trout at the Island of Gotland, Sweden, delineated from mitochondrial DNA. J Fish Biol 60: 49-71.

Landry C, Bernatchez L (2001). Comparative analysis of population structure across environments and geographical scales at major histocompatibility complex and microsatellite loci in Atlantic salmon (Salmo salar). Mol Ecol 10: 2525-2539.

Maruyama T, Nei M (1981). Genetic variability maintained by mutation and overdominant selection in finite populations. Genetics 98: 441-459.

McKay JK, Latta RG (2002). Adaptive population divergence: markers, QTL and traits. Trends Ecol Evol 17: 285-291.

Merilä J, Crnokrak P (2001). Comparison of genetic differentiation at marker loci and quantitative traits. J Evol Biol 14 892-903.

Meyer D, Thomson G (2001). How selection shapes variation of the human major histocompatibility complex: a review. Ann Hum Genet 65: 1-26.

Miller KM, Kaukinen KH, Beacham TD, Withler RE (2001). Geographic heterogeneity in natural selection on an MHC locus in sockeye salmon. Genetica 111: 237.
Monaco JJ (1992). Antigen presentation: not so groovy after all. Curr Biol 2: 433-435.

O'Reilly PT, Hamilton LC, McConnell SK, Wright JM (1996). Rapid analysis of genetic variation in Atlantic salmon (Salmo salar) by PCR multiplexing of dinucleotide and tetranucleotide microsatellite. Can J Fish Aquat Sci 53: 2292-2298

Piertney SB, Oliver MK (2006). The evolutionary ecology of the major histocompatibility complex. Heredity 96: 7-21.

Powis SJ, Deverson EV, Copadwell WJ, Cirula A, Huskisson NS, Smith H et al. (1992). Effect of polymorphism of an MHClinked transporter on the peptides assembled in a class I molecule. Nature 357: 211-215.

R Development Core Team (2006). R: A Language and Environment for Statistical Computing. R Foundation for Statistical Computing: Vienna, Austria. ISBN 3-900051-07-0, URL http:/ / www.R-project.org.

Rajalingam R, Singal DP, Mehra NK (1997). Transporter associated with antigen-processing (TAP) genes and susceptibility to tuberculoid leprosy and pulmonary tuberculosis. Tissue Antigens 49: 168-172.

Raymond M, Rousset F (1995a). An exact test for population differentiation. Evolution 49: 1280-1283.

Raymond M, Rousset F (1995b). GENEPOP (version 1.2) a population genetics software for exact tests and ecumenicism. I Hered 86: 248-249.

Rice WR (1989). Analyzing tables of statistical tests. Evolution 43: 223-225.

Roberts RJ (1993). Ulcerative dermal necrosis (UDN) in wild salmonids. Fish Res 17: 3-14.

Robertson A, Hill WG (1984). Deviations from Hardy-Weinberg proportions: sampling variances and use in estimation of inbreeding coefficients. Genetics 107: 703-718.

Sakamoto T, Danzmann RG, Okamoto N, Ferguson MM, Ihssen PE (1999). Linkage analysis of quantitative trait loci associated with spawning time in rainbow trout (Onchorhynchus mykiss). Aquaculture 173: 33-43.

Schlötterer C (2002). A microsatellite-based multilocus screen for the identification of local selective sweeps. Genetics 160: 753-763.

Slade RW, McCallum HI (1992). Overdominant vs frequencydependent selection at MHC loci. Genetics 132: 861.

Slettan A, Olsaker I, Lie Ø (1995). Atlantic salmon, Salmo salar, microsatellites at the SSOSL25, SSOSL85, SSOSL311, SSOSL417 loci. Anim Genet 26: 281-282.

Slettan A, Olsaker I, Lie Ø (1996). Polymorphic Atlantic salmon, Salmo salar L. microsatellites at the SSOSL438, SSOSL439 and SSOSL444 loci. Anim Genet 27: 57-64.

Storz JF (2005). Using genome scans of DNA polymorphism to infer adaptive population divergence. Mol Ecol 14: 671-688.

Storz JF, Dubach JM (2004). Natural selection drives altitudinal divergence at the albumin locus in deer mice, Peromyscus maniculatus. Evolution 58: 1342-1352.

Taggart JB, Hynes RA, Prodöhl PA, Ferguson A (1992). A simplified protocol for routine total DNA isolation from salmonid fishes. J Fish Biol 40: 963-965.

Takahata N, Nei M (1990). Allelic genealogy under overdominant and frequency-dependent selection and polymorphism of major histocompatibility complex loci. Genetics 124: 967.

Taylor EB (1991). A review of local adaptation in Salmonidae, with particular reference to Pacific and Atlantic salmon. Aquaculture 98: 185-207.

Trowsdale J (1995). Both man and beast: comparative organization of MHC genes. Immunogenetics 41: 1-17.

Vasemägi A, Gross R, Paaver T, Koljonen M-L, Säisä M, Nilsson $\mathrm{J}$ (2005). Analysis of gene associated tandem repeat markers in Atlantic salmon (Salmo salar L.) populations: implications for restoration and conservation in the Baltic sea. Conserv Genet 6: 385-397. 
Wang J, Whitlock MC (2003). Estimating effective population size and migration rates from genetic samples over space and time. Genetics 163: 429-446.

Weir BS (1996). Genetic Data Analysis II. Sinauer Associates: Sunderland, MA.

Weir BS, Cockerham CC (1984). Estimating F-statistics for the analysis of population structure. Evolution 38: 1358-1370.
Westerdahl H, Hansson B, Bensch S, Hasselquist D (2004). Between-year variation of MHC allele frequencies in great reed warblers: selection or drift? J Evol Biol 17: 485-492.

Young FW (1996). Vista: the visual statistics system. Research Memorandum, 94-1 (b) L.L. Thursone Psychometric Laboratory, University of North Carolina: Chapel Hilll, NC.

Supplementary Information accompanies the paper on Heredity website (http://www.nature.com/hdy) 